\title{
Nível de Atividade Física e Tabagismo em Moradores de uma Comunidade em Salvador
}

\author{
Eliane Cerqueira Lima \\ Luciana Bilitário Macedo
}

Tipo de Análise do Manuscrito

Recebido em Nov/ 2011
Fisioterapeuta graduada pela Escola Bahiana

de Medicina e Saúde Pública (EBMSP)

(llanylima@gmail.com)

Fisioterapeuta, Mestre em Medicina e Saúde

Humana. Docente EBMSP e Universidade do

Estado da Bahia (UNEB)

(lucianabilitario@bahiana.edu.br)

Triple Blind Peer Review

Aprovado em Dez/ 2011

\section{RESUMO}

Introdução: 0 tabagismo é um dos maiores problemas de saúde pública e uma das principais causas de mortes evitáveis. Há, portanto, necessidade de um melhor conhecimento de medidas para o controle do uso do tabaco. Objetivo: verificar se existe associação entre atividade física e tabagismo em indivíduos moradores de uma comunidade em Salvador. Método: 43 indivíduos tabagistas adultos foram classificados em três categorias de atividade física (utilizando o questionário internacional de atividade física (IPAQ) e três de tabagismo, utilizando a classificação através do questionário de Fagerström. Resultados: 81,4\% dos participantes foram do sexo masculino; $56 \%$ foram classificados em nível muito ativo de atividade física pelo IPAQ. Indivíduos classificados como muito ativos, apresentaram menor dependência ao fumo $(\mathrm{p}=0,047)$. Conclusões: na amostra houve associação entre maior nível de atividade física e menor dependência ao fumo. Considerando a alta porcentagem de homens e de sujeitos com níveis elevados de atividade física, deve-se exercer cautela ao extrapolar estes resultados para outras populações.

Palavras-chave: tabagismo, atividade física

\section{Physical Activity and Smoking in a Community Residents in Salvador}

\section{ABSTRACT}

Introduction: Smoking is a major public health problems and a major cause of preventable deaths. There is therefore need for a better understanding of measures to control tobacco use. Objective: To investigate the association between physical activity and smoking in individuals living in a community in Salvador. Methods: 43 adult smokers were classified into three categories of physical activity (using the International Physical Activity Questionnaire (IPAQ) and three smoking, using the classification by the Fagerström questionnaire. Results: $81.4 \%$ of participants were sex male, $56 \%$ were classified as very active level of physical activity by IPAQ. Individuals classified as very active, showed less dependence on smoking ( $\mathrm{p}$ $=0.047$ ). Conclusion: the sample was associated with higher levels of physical activity and less dependence on smoking . Considering the high percentage of men and subjects with high levels of physical activity, you should exercise caution in extrapolating these results to other populations.

Keywords: smoking, physical activity

${ }^{1}$ Agradecemos a Carlos Torres Marchal pela ajuda na realização dos cálculos estatísticos. 


\section{Actividad física y tabaquismo \\ en una Residentes de la Comunidad en Salvador}

\section{RESUMEN}

Introducción: El tabaquismo es un problema importante de salud pública y una causa importante de muertes evitables. Por lo tanto, la necesidad de una mejor comprensión de las medidas para controlar el consumo de tabaco. Objetivo: Investigar la asociación entre actividad física y el tabaquismo en las personas que viven en una comunidad en El Salvador. Métodos: 43 fumadores adultos se clasificaron en tres categorías de actividad física (utilizando el Cuestionario Internacional de Actividad Física (IPAQ) y el tabaquismo tres, utilizando la clasificación por el cuestionario de Fagerström Resultados: el 81,4\% de los participantes eran del sexo masculino, 56\% fueron clasificados como nivel muy activa de la actividad física por el IPAQ las personas clasificadas como muy activa, mostraron una menor dependencia de fumar $(p=0,047)$. Conclusión:. la muestra se asocia con mayores niveles de actividad física y una menor dependencia del consumo de tabaco. Teniendo en cuenta el alto porcentaje de los hombres y los sujetos con altos niveles de actividad física, usted debe tener cuidado al extrapolar estos resultados a otras poblaciones.

Palabras clave: tabaquismo, actividad física

\section{L'activité physique et le tabagisme dans un résidents de la communauté à Salvador}

\section{RÉSUMÉ}

Introduction: Le tabagisme est un des problèmes majeurs de santé publique et une cause majeure de décès évitables. Il est donc nécessaire pour une meilleure compréhension des mesures pour contrôler l'usage du tabac. Objectif: étudier l'association entre l'activité physique et le tabagisme chez les personnes vivant dans une communauté à Salvador. Méthodes: 43. Fumeurs adultes ont été classés en trois catégories d'activité physique (en utilisant les International Physical Activity Questionnaire (IPAQ) et trois de fumer, en utilisant la classification par le questionnaire de Fagerström Résultats: $81,4 \%$ des participants étaient le sexe hommes, $56 \%$ ont été classés comme très actif au niveau de l'activité physique par IPAQ personnes classées comme très actif, a montré une moindre dépendance sur le tabagisme $(p=0,047)$. Conclusion:. l'échantillon a été associée à des niveaux plus élevés d'activité physique et moins de dépendance sur le tabagisme. Compte tenu du pourcentage élevé d'hommes et les sujets ayant des niveaux élevés d'activité physique, vous devriez faire preuve de prudence dans l'extrapolation de ces résultats à d'autres populations.

Mots-clés: tabagisme, activité physique

\section{Introdução}

O tabagismo é considerado pela Organização Mundial de Saúde (OMS) como a principal causa de doenças e morte evitável e um dos maiores problemas de saúde pública de proporções mundiais. 0 consumo de tabaco pode causar dependência física, psicológica ou emocional e dependência comportamental ou de hábito.1,2

A nicotina é a substância psicoativa presente no tabaco que provoca a dependência química, similar a dependência a drogas como heroína e cocaína. As ações psíquicas da nicotina são complexas, com uma mistura de efeitos estimulantes e depressores. Menciona-se o aumento da concentração e da atenção e a redução do apetite e da ansiedade em fumantes. A nicotina também induz tolerância e provoca síndrome de abstinência com alteração do 
sono, irritabilidade, diminuição da concentração e ansiedade. Além disso, aumenta a liberação de catecolaminas, causa vasoconstricção, acelera a freqüência cardíaca, causando hi-pertensão arterial 1,2.

A nicotina, juntamente com o monóxido de carbono, provocam diversas doenças cardiovasculares, bem como, estimulam no aparelho gastrointestinal a produção de ácido clorídrico, que pode ocasionar a úlcera gástrica. Outro efeito prejudicial, é a liberação de substâncias quimiotáxicas no pulmão, estimulando um processo que irá destruir a elastina, provocando o enfisema pulmonar.2

No mundo e no Brasil o tabagismo vem se concentrando cada vez mais em populações de menor escolaridade e renda. Diante deste cenário, 192 países membros da OMS, na Assembleia Mundial de Saúde realizada em maio de 2003, assinaram a Convenção Quadro para Controle do Tabaco (CQCT), primeiro tratado internacional de Saúde Pública, negociado através da OMS assumindo o compromisso da implementação de medidas intersetoriais para o controle do tabaco. A adesão do Brasil a este tratado foi ratificada pelo Congresso Nacional em novembro de 2005.2,3

A atividade física é um comportamento complexo influenciado por vários fatores como: idade, sexo, educação e nível socioeconômico.1,2,3,4, Pode ser definida como qualquer movimento corporal produzido pelos músculos esqueléticos que resulte em gasto energético. Seus benefícios são amplamente apresentados e discutidos na literatura.1,2,3,4,5 Entretanto são escassos os estudos que indicam a atividade física como medida eficaz de intervenção em indivíduos fumantes.

Este trabalho teve como objetivo verificar se existe associação entre o nível de atividade física e tabagismo em moradores de uma comunidade em Salvador.

\section{Material \& Método}

Trata-se de um estudo de corte transversal, realizado com um subgrupo de participantes do projeto Tabagismo, Força Muscular e Atividade Física em Adultos, realizado na Escola Bahiana de Medicina e Saúde Pública em Salvador, Bahia.

A pesquisa foi conduzida em uma comunidade no bairro da Boca do Rio, na cidade de Salvador, Bahia, em 2010.

A amostra foi composta por 43 indivíduos tabagistas, com idade igual ou superior a 18 anos, ambos os gêneros. Foram excluídos indivíduos ex-fumantes, gestantes e com doenças cardiovasculares e respiratórias crônicas prévias.

A coleta de dados foi realizada através de avaliação por meio de uma ficha simplificada possuindo os itens: sexo, idade, presença ou não de doenças prévias e antecedentes familiares, medicações em uso. As medições de freqüência cardíaca (FC), pressão arterial sistólica (PAS) e pressão arterial diastólica (PAD) foram realizadas por dois examinadores treinados. Após cinco minutos de repouso, com o participante sentado e braço direito posicionado à altura do coração, foi aferida a PAS e PAD e a FC através de um esfigmomanômetro digital (OMRON) modelo HEM-711 em um único momento.

A atividade física diária foi determinada pelo questionário internacional de atividade física (IPAQ), versão cur-ta, que classifica o nível de atividade física, por meio de uma pontuação obtida pela soma da quantidade de dias e minutos ou horas de atividades físicas realizadas nas duas semanas anteriores ao preenchimento do questionário. $(5,6)$ Considerando os critérios de freqüência, intensidade e duração, os níveis de atividade física foram classificados em: pouco ativos, moderadamente ativos e muito ativos. Foi também estimado o gasto energético metabólico considerando os minutos por semana para cada atividade em equivalentes metabólicos (METS/ min/sem).(5,6,7).

0 grau de dependência nicotínica foi avaliado pelo teste de dependência à nicotina de Fagerström, que consiste em seis questões sobre freqüência, quantidade e necessidade de 
consumir tabaco. Por meio das respostas com pontuações específicas, os participantes foram classificados em dependência leve, moderada e grave. $(7,8)$

Para análise estatística foi utilizado o programa Statistical Package for the Social Sciences (SPSS) versão 16.0 para Windows licenciado para a Escola Bahiana de Medicina e Saúde Pública. As variáveis numéricas foram expressas em medidas de tendência central após ser avaliada a normalidade das mesmas. As variáveis categóricas foram expressas em frequências absolutas, e foi aplicado o teste exato de Fisher.9,10 Em todas as análises adotouse $5 \%$ como nível de significância.

O estudo foi aprovado pelo Comitê de Ética em Pesquisa da Faculdade de Tecnologia e Ciências, parecer número 1992, em 09 de agosto de 2010.

\section{Resultados}

0 estudo contou com uma amostra de 43 fumantes, sendo a maioria (35) do sexo masculino com 81,4\%. A média de idade foi de 38,5 anos (DP $\pm 13,03$ ). A FC, PAS, PAD apresentam valores médios dentro da normalidade (Tabela 1).

Tabela 1

Características da amostra de fumantes quanto à idade, equivalentes metabólicos e variáveis fisiológicas

\begin{tabular}{lc|c|c}
\hline $\begin{array}{c}\text { Variáveis } \\
(\mathrm{N}=43)\end{array}$ & $\begin{array}{c}\text { Média } \\
\text { (Em anos) }\end{array}$ & $\begin{array}{c}\text { Valores Mínimos } \\
\text { e Máximos }\end{array}$ & Desvio Padrão \\
\hline IDADE (anos) & 38,56 & $(18+-66)$ & 13,03 \\
FC (bpm) & 78,53 & $(55+1110)$ & 12,47 \\
PAS (mmHg) & 120,35 & $(90+\dashv 180)$ & 19,16 \\
PAD (mmHg) & 79,30 & $(60 \vdash-120)$ & 13,16 \\
METS (METS/min/sem) & $2719 *$ & $(0+-21.546)$ & 5549,30 \\
\hline
\end{tabular}

OBS: Variáveis numéricas expressas em medidas de tendência central. FC, freqüência cardíaca; PAS,

Pressão arterial sistólica; PAD, Pressão arterial diastólica; METS, equivalentes metabólicos avaliados pelo IPAQ. * Mediana

Quanto ao nível de atividade física, 55\% (24) dos tabagistas foram classificados como muito ativos; $24 \%$ (10) como moderadamente ativos e $21 \%$ (9) como pouco ativos. Em relação ao grau de dependência $20(46,5 \%)$ foram classificados como com leve dependência, 11 (25,5\%) moderada dependência e 12(18\%) como com grave dependência. Quando comparados o nível de atividade física com o grau de dependência ao tabagismo, a maioria 24 $(55 \%)$ dos sujeitos foi classificada como muito ativos e apresentaram uma maior freqüência 62,5\%(15) de indivíduos com leve dependência em relação aos demais grupos. Indivíduos classificados como muito ativos, apresentaram menor dependência ao fumo $(p=0,047)$.

Tabela 2

Nível de atividade física (IPAQ) e grau de dependência ao fumo (Fargestrom)

\begin{tabular}{l|c|c|c|c}
\hline \multirow{2}{*}{ IPAQ } & \multicolumn{3}{c|}{ Dependência ao Fumo } & \multirow{2}{*}{ Total } \\
\cline { 2 - 4 } & Leve & Moderada & Grave & \\
\hline Pouco ativos & $11,1 \%(1)$ & $44,4 \%(4)$ & $44,4 \%(4)$ & $21 \%(9)$ \\
Moderadamente ativos & $40 \%(4)$ & $40 \%(4)$ & $20 \%(2)$ & $24 \%(10)$ \\
Muito ativos & $62,5 \%(15)$ & $12,5 \%(3)$ & $25 \%(6)$ & $55 \%(24)$ \\
\hline
\end{tabular}

OBS: IPAQ = Questionário Internacional de Atividade Física. 


\section{Discussão}

Neste estudo foi encontrado um maior número de indivíduos fumantes do sexo masculino. Isto é confirmado por dados disponibilizados pelo Instituto Nacional do Câncer (INCA, 2010) que indica que $47 \%$ de toda a população masculina e $12 \%$ da população feminina no mundo fumam, sugerindo que os homens fumam mais do que as mulheres, apesar de uma tendência crescente do número de mulheres fumantes11.

Os valores de PAS, PAD e FC mostraram-se dentro do esperado para indivíduos na população geral. 0 artigo de Pureza et al. (2007) 12 comparou estas mesmas variáveis entre mulheres tabagistas e não tabagistas, concluindo que a PAD e a FC foram maiores nas mulheres fumantes quando comparadas com as mulheres não fumantes e a PAS foi similar em ambos os grupos. Outro estudo13 evidenciou que fumando um único cigarro, a FC eleva-se até $10 / 20$ batimentos/minutos e esse aumento pode regredir após 15/45 minutos. Estes resultados não foram avaliados na presente pesquisa e não cabe comparação devido o público maior neste, ser de homens e não ser avaliada a FC imediatamente após o ato tabágico.

Ao avaliar a correlação entre níveis de atividade física e tabagismo, foi encontrada uma relação inversa entre as categorias de atividade física muito ativa e grau de dependência ao fumo leve. Em relação aos outros grupos não foi observado uma tendência muito clara com o grau de dependência à nicotina. A provável explicação para este resultado pode estar relacionada à alta porcentagem de sujeitos com níveis muito ativos de atividade física avaliados pelo IPAQ, e de homens que exerciam funções e ocupações que exigiam grande esforço, de acordo com os relatos descritos durante a coleta, porém, não coletados na ficha de avaliação.

Ao analisar as proporções entre os indivíduos com prática de atividade física muito ativa em relação às outras categorias, observou-se a seguinte relação: 2,4 para cada 1 que tenha alegado a prática moderada ou moderadamente ativos; 2,6 para cada classificado com nível leve ou inativos. Supõe-se que o elevado número de sujeitos com dependência leve devese a esta proporção, dado o fato que 15 dos 20 pacientes com este nível de tabagismo se encontram nesta categoria de atividade física ou muito ativos. Essa relação não foi observada entre os indivíduos classificados como inativos pelo IPAQ, em que houve uma maioria 4 $(44,4 \%)$ de dependentes graves a nicotina, o que sugere uma espécie de proporção inversa entre níveis de atividade física e tabagismo.

Observou-se também que houve um predomínio do grau leve de dependência ao tabaco. Esses achados corroboram com os resultados de outros autores.8,14-17 Além disso, em estudo realizado em universitários, com o objetivo de avaliar o nível de atividade física nesse grupo e sua relação com o fumo, verificou-se que o grau de dependência ao fumo muito leve foi mais prevalente $(68,5 \%, p<0,05)$. Esses achados supõem que o ambiente e os aspectos climáticos, associados à atuação de programas nacionais de intervenção contra o tabagismo favorecem a prática de atividade física e diminuem o grau de dependência ao fumo.

Em uma revisão de literatura18 foi encontrada uma avaliação de 50 artigos relacionados ao tema, sendo que $60 \%$ destes trabalhos relataram uma associação inversa entre tabagismo e atividade física. Contudo esta relação foi freqüentemente atenuada ou revertida entre adolescentes e homens, no caso de praticantes de exercícios moderado, em contraposição a exercícios vigorosos.

Em contrapartida outro artigo19 de revisão que analisou mais de 40 trabalhos, concluiu que há uma relação invertida entre tabagismo e atividade física não laboral, ou seja, menor atividade física esteve associada a maior dependência ao fumo. Esses resultados sugerem que alguns fatores socioeconômicos e de ordem biológica como, por exemplo, idade, sexo e raça possam influenciar na resposta ao exercício e nos níveis de intensidade.

Neste trabalho os indivíduos muito ativos, tiveram menor grau de dependência ao fumo. Comparando os níveis de intensidade de exercício na literatura17, 18, 20 visando verificar seu 
efeito sobre o desejo de fumar e a abstinência, encontrou-se que pessoas com níveis de atividade física de moderada a vigorosa tendem a aumentar o tempo em relação ao desejo de fumar e atenuar os sintomas de abstinência.

Adicionalmente, outros autores17, 18 demonstraram que a prática de atividades físicas vigorosas tendem a facilitar que o indivíduo deixe de fumar, em curto ou longo prazo, quando combinado com estratégias cognitivas, além de apresentar evolução na capacidade física geral do praticante.

Desta forma, ressalta-se a importância do treinamento físico para minimizar as implicações nutricionais atribuídas ao abandono do tabagismo, principalmente os relacionados ao aumento de peso. Assim, parece que a atividade física apresenta um papel protetor contra o tabagismo, embora não esteja elucidado o mecanismo pelo qual isso acontece, sendo um tema com lacunas a serem futuramente preenchidas.

\section{Conclusão}

Neste artigo, que visou verificar se há relação entre nível de atividade física e tabagismo, foi encontrada uma relação inversa entre as variáveis estudadas. Isto sugere que a atividade física exerça efeitos positivos na prevenção e no tratamento do tabagismo.

Foram limitações deste estudo: o tamanho amostral (43 indivíduos), a maior proporção de homens $(81,4 \%)$ em relação às mulheres; a avaliação dos níveis de atividade física pelo IPAQ, bem como a avaliação do grau de dependência ao fumo pelo Fargestrom de forma categórica apenas, limitando as análises, não permitindo as correlações entre as variáveis numéricas. Outra limitação foi a não avaliação da carga tabágica dos indivíduos, visto que o tempo de consumo está relacionado a degradação do sistema cardiovascular e pulmonar.

\section{Referências}

1. DUARTE, P. Prevenção ao uso de álcool e outras drogas no ambiente de trabalho. Florianópolis: Universidade Federal de Santa Catarina, 2008.

2. ROSEMBERG, J; ROSEMBERG, A; MORAES, M. A. Nicotina droga universal. Rio de Janeiro: INCA, 2008. p. 239.

3. INCA - Instituto Nacional do Câncer. A história do tabaco. Brasília, Brasil, 2009. Disponível em: http://www.inca.gov.br/tabagismo/ (Acesso em novembro de 2011).

4. UMEDA, I. I. K. Fisioterapia e fatores de risco da doença cardiovascular. Barueri SP: Manole, p40. 2005.

5. BENEDETTI, T. R. et al. "Reprodutibilidade e validade do Questionário Internacional de Atividade Física (IPAQ) em homens idosos”, Florianópolis-SC: Revista Brasileira de Medicina do Esporte, 13(1): Jan/ Fev 2007.

6. Centro de Estudos do Laboratório de Aptidão Física de São Caetano do Sul - CELAFISCS. Questionário internacional de atividade física - Versão curta. Disponível em: www celafiscs.institucional.ws/81/ questiona-rios/ipaq_curto.html. (Acesso em: Fev/2010).

7. PIETROBON, R. C. et al. "Utilização do teste de dependência à nicotina de Fagerström como um instrumento medida do grau de dependência”, Porto Alegre-RS: Revista HCPA, 27(3): 31-36. 2007.

8. RODRIGUES, E. S. et al. "Nível de atividade física e tabagismo em universitários", Revista de Saúde Pública, São Paulo, 42(4): 672-78. Ago/ 08.

9. LOWRY, R. Fisher "Exact Probability Test: 3x3", Disponível em: http://faculty.vassar.edu/ lowry/fisher3x3.html. (Acesso em 19/06/2011).

10. FREEMAN, G.H; HALTON, J. H. "Note on exact treatment of contingency, goodness of fit and other problems of significance”, Biometrika, 38(1-2): 141-49. Jun/ 51.

11. INCA - Instituto Nacional do Câncer. Tabagismo no Brasil. Disponível em: http:// www.inca.gov.br/ tabagismo/frameset.asp?item=dadosnum\&link=mundo.htm. (Acesso em: Nov-2011). 
12. PUREZA DY, SARGENTINI L, LATERZA R, FLORES LJF et. al. "Efeitos cardiovasculares da abstinência do fumo no repouso e durante o exercício submáximo em mulheres jovens fumantes", Rev Bras Med Esporte, 13(5): 292-96. 2007.

13. HOLLMANN W, HEITTINGER T H. Medicina de esporte: fumo e capacidade de desempenho físico. São Paulo: Manole, 1989.

14. BOUTELLE, K. N. et al. "Associations between Exercise and Health Behaviors in a Community Sample of Working Adults", Amsterdam: Preventive Medicine, 30(3): 217-24. Mar/ 00.

15. PETERS, T. M. “Accelerometer-Measured Physical Activity in Chinese Adults”, Amsterdam: American Journal of Preventive Medicine, 38(6): 583-91. 2010.

16. MARCUS B H; ALBRECHT A E; NIAURA R S; ABRAMS D B; THOMPSON P D. “Usefulness of physical exercise for maintaining smoking cessation in women", American Journal of Cardiology, (68): 40607.1991.

17. TAOYLOR A H; USSHER M H; FAULKNER G. "The acute effects of exercise on cigarette cravings, with-drawal symptoms, affect and smoking behaviour: a systematic review", 534-43. 2007.

18. KACZYNSKI, A. T. et al. "Smoking and Physical Activity: a Systematic review", Oak Ridge: American Journal of Health Behavior, 32(1): 93-110. 2008.

19. BLAIR, S. N.; JACOBS, D. R.; POWELL, K. E. “Relationships between exercise of physical activity and other health behaviors", Public Health Reports, 100(2): 172-80. Mar-Abr/ 85.

20. EVERSON ES, DALEY AJ, USSHER M. "Moderate and vigorous intensity exercise acutely reduce cravings and withdrawal symptoms in abstaining young adult smokers", Mental Health and Physical Activity, p. 26.2008. 